\title{
Conversions of parts per million on soil test reports to pounds per acre ${ }^{1}$
}

\author{
Guodong Liu, Yuncong Li, and Aparna Gazula²
}

Soil testing and the resulting fertilization recommendations are critical for appropriate nutrient management in commercial vegetable production. It is just as critical that test results and fertilization recommendations are understood by growers. UF/IFAS soil test reports use parts per million (ppm) to report soil-extractable plant nutrients. The UF/ IFAS fertilizer recommendations use pounds of nutrient per acre. Growers also operate with pounds of a particular nutrient per acre to apply fertilizers. If growers don't understand the soil test reports and fertilization recommendations, however, they may over-fertilize crops. One way to avoid miscommunication is to remember that growers and soil experts sometimes speak different languages.

Nutrients in soil can be classified in several ways (water-soluble nutrients, extractable nutrients, etc.). For fertilization recommendations, soil labs usually use the term, extractable nutrients. The level of extractable nutrients in a soil sample is determined by the extractant used. This means that when we talk about soil nutrient contents, we need to know what extractant was used to obtain the sample because different extractants can give totally different values even for the same soil sample. For example, using a particular extractant (e.g. Mehlich-I) to extract a soil sample can obtain a value of a specific nutrient such as phosphorus, e.g., $50 \mathrm{ppm}$. This value means that the tested soil contains $50 \mathrm{mg}$ of MehlichI-extractable phosphorus per $\mathrm{kg}$ of soil. The phosphorus level of a particular soil sample will be greater if the extractant Mehlich-I was used than the phosphorus level in the same sample if the extractant Mehlich-III, Bray-1, or Olsen was used. If no extractant is listed on the soil test report, the reported phosphorus level will be relatively meaningless.

The terms extractant and extractable phosphorus are commonly used and understood in soil labs, but may not be understood as well by growers. To avoid misunderstandings and over- or under-fertilization, we must understand what relationship exists between the numbers of soil test reports, fertilization recommendations, and fertilization practices. How can we compare these numbers? The conversion from parts per million of soil-extractable nutrients on a soil test report to pounds per acre will be helpful for producers. The conversion can, in turn, help to (1) better understand how much extractable nutrient is in the soil; (2) optimize fertilization practices based on these reports; and (3) minimize over-fertilization through optimization of fertilization.

If our soils contain very high levels of phosphorus and calcium but phosphate fertilizers and gypsum are still applied every year, what will happen? The answer is that these applications do not only waste nonrenewable resources but also produce phosphate rock in the fertilized field, particularly when soil $\mathrm{pH}$ is greater than 6.5. For example, applying 100 to $120 \mathrm{lb}_{2} \mathrm{O}_{5}$ per acre is not only unnecessary but also harmful to the environment if a calibrated soil test shows a very high level (e.g., $550 \mathrm{ppm}$ ) of phosphorus existing in the soil. Similarly, $1000 \mathrm{lb}$ gypsum (233 lb calcium) per acre every year should not be applied if the soil test shows

1. This document is HS1229, one of a series of the Horticultural Sciences Department, UF/IFAS Extension. Original publication date August 2013. Visit the EDIS website at http://edis.ifas.ufl.edu.

2. Guodong Liu, assistant professor, Horticultural Sciences Department, UF/IFAS, 1253 Fifield Hall, Gainesville, FL 32611; Yuncong Li, professor, Soil and Water Science Department, Tropical Research and Education Center, UF/IFAS, 18905 SW 280th Street, Homestead, FL 33031; Aparna Gazula, UF/IFAS Alachua County Extension, 2800 NW 39 Ave, Gainesville, FL 32609. 
a very high level of calcium (e.g., $1550 \mathrm{ppm})$. This article provides a simple conversion method for crop consultants, crop advisors, growers, students, and researchers who are interested in nutrient and water management of crop production.

\section{How to convert parts per million to pounds per acre}

If we assume the plow layer or root zone depth is 6 inches, the total weight of soils in the plow layer can be calculated as:

Soil weight per acre $=$ volume $\mathrm{x}$ bulk density

$=\left(43560 \mathrm{ft}^{2} / \mathrm{acre} \times 0.5 \mathrm{ft}\right) \times 92 \mathrm{lb} / \mathrm{ft}^{3} \approx 2,000,000 \mathrm{lb} / \mathrm{acre}$

Because UF/IFAS soil test reports report extractable nutrients in parts per million, we can readily use a factor of 2 to convert the actual number of ppm to pounds of a particular extractable nutrient per acre. In the above example, the extractable concentrations were reported as $550 \mathrm{ppm}$ of phosphorus and $1550 \mathrm{ppm}$ of calcium. These numbers mean that soil has 1100 pounds of extractable phosphorus per acre and 3100 pounds of calcium per acre. Further, in fertilization of phosphorus and potassium, their oxides are used. Therefore, the pounds of extractable phosphorus pentoxide $\left(\mathrm{P}_{2} \mathrm{O}_{5}\right)$ and potassium oxide $\left(\mathrm{K}_{2} \mathrm{O}\right)$ need to be calculated by additional conversion factors of 2.2913 and 1.2046 for phosphorus and potassium, respectively. The soil in the above example has $550 \mathrm{ppm}$ phosphorus and hence contains 2520 pounds of extractable phosphorus pentoxide per acre. The conversion equations are below. More conversions are available in Table 1.

Extractable nutrient $(\mathrm{ppm}) \times 2=$ pounds of the extractable nutrient per acre

Extractable phosphorus $(\mathrm{ppm}) \times 2 \times 2.2913=$ pounds of the extractable phosphorus pentoxide per acre

Extractable potassium $(\mathrm{ppm}) \times 2 \times 1.2046=$ pounds of the extractable potassium oxide per acre

Of course, the amount extracted by the soil test is not directly equivalent to fertilizer grade nutrients, and the soil chemistry also influences the plant-available nutrient. Since the soil test is used to predict crop response to added nutrients, at these large soil-test values, we can expect little plant response to application of fertilizers. We must consider other actions and management decisions that can create a confusing and inefficient use of nutrients. The right amount of phosphorus and gypsum (calcium) from fertilizer can help plants grow, but too much will form rock phosphate and related compounds, none of which nutrient sources are available to plants. You will find more information on this and related issues in forthcoming EDIS documents.

\section{How to convert pounds per acre to parts per million:}

We can easily use a factor of 0.5 to convert the pounds per acre of a nutrient in its elemental form into ppm. To convert 120 pounds per acre to ppm, just multiply 120 pounds per acre by 0.5 , which is to equal $60 \mathrm{ppm}$. This conversion works for all nutrients reported in these units: macronutrients, such as calcium or magnesium, and micronutrients, such as iron, manganese, zinc, and copper. Again, another factor is needed when converting pounds per acre of phosphorus and potassium in their oxide forms into number of ppm in their elemental forms: 0.4364 and 0.8302 , respectively. For example, to convert 100 pounds per acre of phosphate fertilizer $\left(\mathrm{P}_{2} \mathrm{O}_{5}\right)$ to ppm of elemental phosphorus, just multiply 100 by 0.5 and then by 0.4364 to get $21.8 \mathrm{ppm}$ of phosphorus. Similarly, 100 pounds per acre of potash fertilizer $\left(\mathrm{K}_{2} \mathrm{O}\right)$ will increase soil potassium (K) by $41.5 \mathrm{ppm}$. The conversion equations are below. More conversions are available in Table 2.

Pounds of nutrient applied per acre $\times 0.5=$ increment of the nutrient in soil (ppm)

Pounds of phosphorus pentoxide applied per acre $\times 0.5 \times$ $0.4364=$ increment of phosphorus in soil $(\mathrm{ppm})$

Pounds of potassium oxide applied per acre $\times 0.5 \times 0.8302=$ increment of potassium in soil (ppm) 
Table 1. Conversion from ppm on soil test report to pounds per acre for fertilization practices

\begin{tabular}{|c|c|c|c|c|c|}
\hline \multirow{2}{*}{$\begin{array}{l}\text { Soil test } \\
\text { PPM }\end{array}$} & \multicolumn{5}{|c|}{ Pounds per acre } \\
\hline & $\mathrm{P}$ & $\mathrm{P}_{2} \mathrm{O}_{5}$ & $\mathrm{~K}$ & $\mathrm{~K}_{2} \mathrm{O}$ & Other nutrients \\
\hline 5 & 10 & 22.9 & 10 & 12.0 & 10 \\
\hline 10 & 20 & 45.8 & 20 & 24.1 & 20 \\
\hline 15 & 30 & 68.7 & 30 & 36.1 & 30 \\
\hline 20 & 40 & 91.7 & 40 & 48.2 & 40 \\
\hline 25 & 50 & 114.6 & 50 & 60.2 & 50 \\
\hline 30 & 60 & 137.5 & 60 & 72.3 & 60 \\
\hline 35 & 70 & 160.4 & 70 & 84.3 & 70 \\
\hline 40 & 80 & 183.3 & 80 & 96.4 & 80 \\
\hline 45 & 90 & 206.2 & 90 & 108.4 & 90 \\
\hline 50 & 100 & 229.1 & 100 & 120.5 & 100 \\
\hline 55 & 110 & 252.0 & 110 & 132.5 & 110 \\
\hline 60 & 120 & 275.0 & 120 & 144.6 & 120 \\
\hline 65 & 130 & 297.9 & 130 & 156.6 & 130 \\
\hline 70 & 140 & 320.8 & 140 & 168.6 & 140 \\
\hline 75 & 150 & 343.7 & 150 & 180.7 & 150 \\
\hline 80 & 160 & 366.6 & 160 & 192.7 & 160 \\
\hline 85 & 170 & 389.5 & 170 & 204.8 & 170 \\
\hline 90 & 180 & 412.4 & 180 & 216.8 & 180 \\
\hline 95 & 190 & 435.3 & 190 & 228.9 & 190 \\
\hline 100 & 200 & 458.3 & 200 & 240.9 & 200 \\
\hline 105 & 210 & 481.2 & 210 & 253.0 & 210 \\
\hline 110 & 220 & 504.1 & 220 & 265.0 & 220 \\
\hline 115 & 230 & 527.0 & 230 & 277.1 & 230 \\
\hline 120 & 240 & 549.9 & 240 & 289.1 & 240 \\
\hline 125 & 250 & 572.8 & 250 & 301.2 & 250 \\
\hline 130 & 260 & 595.7 & 260 & 313.2 & 260 \\
\hline 135 & 270 & 618.7 & 270 & 325.2 & 270 \\
\hline 140 & 280 & 641.6 & 280 & 337.3 & 280 \\
\hline 145 & 290 & 664.5 & 290 & 349.3 & 290 \\
\hline 150 & 300 & 687.4 & 300 & 361.4 & 300 \\
\hline 155 & 310 & 710.3 & 310 & 373.4 & 310 \\
\hline 160 & 320 & 733.2 & 320 & 385.5 & 320 \\
\hline 165 & 330 & 756.1 & 330 & 397.5 & 330 \\
\hline 170 & 340 & 779.0 & 340 & 409.6 & 340 \\
\hline 175 & 350 & 802.0 & 350 & 421.6 & 350 \\
\hline 180 & 360 & 824.9 & 360 & 433.7 & 360 \\
\hline 185 & 370 & 847.8 & 370 & 445.7 & 370 \\
\hline 190 & 380 & 870.7 & 380 & 457.7 & 380 \\
\hline 195 & 390 & 893.6 & 390 & 469.8 & 390 \\
\hline 200 & 400 & 916.5 & 400 & 481.8 & 400 \\
\hline 250 & 500 & 1145.7 & 500 & 602.3 & 500 \\
\hline 300 & 600 & 1374.8 & 600 & 722.8 & 600 \\
\hline 350 & 700 & 1603.9 & 700 & 843.2 & 700 \\
\hline 400 & 800 & 1833.0 & 800 & 963.7 & 800 \\
\hline
\end{tabular}




$\begin{array}{llllll}450 & 900 & 2062.2 & 900 & 1084.1 & 900 \\ 500 & 1000 & 2291.3 & 1000 & 1204.6 & 1000 \\ 600 & 1200 & 2749.6 & 1200 & 1445.5 & 1200 \\ 700 & 1400 & 3207.8 & 1400 & 1686.4 & 1400 \\ 800 & 1600 & 3666.1 & 1600 & 1927.4 & 1600 \\ 900 & 1800 & 4124.3 & 1800 & 2168.3 & 1800 \\ 1000 & 2000 & 4582.6 & 2000 & 2409.2 & 2000\end{array}$

Footnote: phosphorus pentoxide $\left(\mathrm{P}_{2} \mathrm{O}_{5}\right)$ contains $43.64 \%$ of phosphorus; potassium oxide $\left(\mathrm{K}_{2} \mathrm{O}\right)$ contains $83.02 \%$ potassium. 
Table 2. Conversion from pounds per acre for fertilization practices to ppm on soil test reports

\begin{tabular}{|c|c|c|c|c|c|}
\hline \multirow{2}{*}{$\begin{array}{l}\text { Soil test } \\
\text { Pounds/acre }\end{array}$} & \multicolumn{5}{|l|}{ PPM } \\
\hline & $\mathrm{P}$ & $\mathrm{P}_{2} \mathrm{O}_{5}$ & $\mathrm{~K}$ & $\mathrm{~K}_{2} \mathrm{O}$ & Other nutrients \\
\hline 1 & 0.5 & 1.1 & 0.5 & 0.6 & 0.5 \\
\hline 2 & 1.0 & 2.3 & 1.0 & 1.2 & 1.0 \\
\hline 3 & 1.5 & 3.4 & 1.5 & 1.8 & 1.5 \\
\hline 4 & 2.0 & 4.6 & 2.0 & 2.4 & 2.0 \\
\hline 5 & 2.5 & 5.7 & 2.5 & 3.0 & 2.5 \\
\hline 6 & 3.0 & 6.9 & 3.0 & 3.6 & 3.0 \\
\hline 7 & 3.5 & 8.0 & 3.5 & 4.2 & 3.5 \\
\hline 8 & 4.0 & 9.2 & 4.0 & 4.8 & 4.0 \\
\hline 9 & 4.5 & 10.3 & 4.5 & 5.4 & 4.5 \\
\hline 10 & 5.0 & 11.5 & 5.0 & 6.0 & 5.0 \\
\hline 11 & 5.5 & 12.6 & 5.5 & 6.6 & 5.5 \\
\hline 12 & 6.0 & 13.7 & 6.0 & 7.2 & 6.0 \\
\hline 13 & 6.5 & 14.9 & 6.5 & 7.8 & 6.5 \\
\hline 14 & 7.0 & 16.0 & 7.0 & 8.4 & 7.0 \\
\hline 15 & 7.5 & 17.2 & 7.5 & 9.0 & 7.5 \\
\hline 16 & 8.0 & 18.3 & 8.0 & 9.6 & 8.0 \\
\hline 17 & 8.5 & 19.5 & 8.5 & 10.2 & 8.5 \\
\hline 18 & 9.0 & 20.6 & 9.0 & 10.8 & 9.0 \\
\hline 19 & 9.5 & 21.8 & 9.5 & 11.4 & 9.5 \\
\hline 20 & 10.0 & 22.9 & 10.0 & 12.0 & 10.0 \\
\hline 21 & 10.5 & 24.1 & 10.5 & 12.6 & 10.5 \\
\hline 22 & 11.0 & 25.2 & 11.0 & 13.2 & 11.0 \\
\hline 23 & 11.5 & 26.4 & 11.5 & 13.9 & 11.5 \\
\hline 24 & 12.0 & 27.5 & 12.0 & 14.5 & 12.0 \\
\hline 25 & 12.5 & 28.6 & 12.5 & 15.1 & 12.5 \\
\hline 26 & 13.0 & 29.8 & 13.0 & 15.7 & 13.0 \\
\hline 27 & 13.5 & 30.9 & 13.5 & 16.3 & 13.5 \\
\hline 28 & 14.0 & 32.1 & 14.0 & 16.9 & 14.0 \\
\hline 29 & 14.5 & 33.2 & 14.5 & 17.5 & 14.5 \\
\hline 30 & 15.0 & 34.4 & 15.0 & 18.1 & 15.0 \\
\hline 31 & 15.5 & 35.5 & 15.5 & 18.7 & 15.5 \\
\hline 32 & 16.0 & 36.7 & 16.0 & 19.3 & 16.0 \\
\hline 33 & 16.5 & 37.8 & 16.5 & 19.9 & 16.5 \\
\hline 34 & 17.0 & 39.0 & 17.0 & 20.5 & 17.0 \\
\hline 35 & 17.5 & 40.1 & 17.5 & 21.1 & 17.5 \\
\hline 36 & 18.0 & 41.2 & 18.0 & 21.7 & 18.0 \\
\hline 37 & 18.5 & 42.4 & 18.5 & 22.3 & 18.5 \\
\hline 38 & 19.0 & 43.5 & 19.0 & 22.9 & 19.0 \\
\hline 39 & 19.5 & 44.7 & 19.5 & 23.5 & 19.5 \\
\hline 40 & 20.0 & 45.8 & 20.0 & 24.1 & 20.0 \\
\hline 41 & 20.5 & 47.0 & 20.5 & 24.7 & 20.5 \\
\hline 42 & 21.0 & 48.1 & 21.0 & 25.3 & 21.0 \\
\hline 43 & 21.5 & 49.3 & 21.5 & 25.9 & 21.5 \\
\hline 44 & 22.0 & 50.4 & 22.0 & 26.5 & 22.0 \\
\hline
\end{tabular}




\begin{tabular}{|c|c|c|c|c|c|}
\hline 45 & 22.5 & 51.6 & 22.5 & 27.1 & 22.5 \\
\hline 46 & 23.0 & 52.7 & 23.0 & 27.7 & 23.0 \\
\hline 47 & 23.5 & 53.8 & 23.5 & 28.3 & 23.5 \\
\hline 48 & 24.0 & 55.0 & 24.0 & 28.9 & 24.0 \\
\hline 49 & 24.5 & 56.1 & 24.5 & 29.5 & 24.5 \\
\hline 50 & 25.0 & 57.3 & 25.0 & 30.1 & 25.0 \\
\hline 51 & 25.5 & 58.4 & 25.5 & 30.7 & 25.5 \\
\hline 52 & 26.0 & 59.6 & 26.0 & 31.3 & 26.0 \\
\hline 53 & 26.5 & 60.7 & 26.5 & 31.9 & 26.5 \\
\hline 54 & 27.0 & 61.9 & 27.0 & 32.5 & 27.0 \\
\hline 55 & 27.5 & 63.0 & 27.5 & 33.1 & 27.5 \\
\hline 56 & 28.0 & 64.2 & 28.0 & 33.7 & 28.0 \\
\hline 57 & 28.5 & 65.3 & 28.5 & 34.3 & 28.5 \\
\hline 58 & 29.0 & 66.5 & 29.0 & 34.9 & 29.0 \\
\hline 59 & 29.5 & 67.6 & 29.5 & 35.5 & 29.5 \\
\hline 60 & 30.0 & 68.7 & 30.0 & 36.1 & 30.0 \\
\hline 61 & 30.5 & 69.9 & 30.5 & 36.7 & 30.5 \\
\hline 62 & 31.0 & 71.0 & 31.0 & 37.3 & 31.0 \\
\hline 63 & 31.5 & 72.2 & 31.5 & 37.9 & 31.5 \\
\hline 64 & 32.0 & 73.3 & 32.0 & 38.5 & 32.0 \\
\hline 65 & 32.5 & 74.5 & 32.5 & 39.1 & 32.5 \\
\hline 66 & 33.0 & 75.6 & 33.0 & 39.7 & 33.0 \\
\hline 67 & 33.5 & 76.8 & 33.5 & 40.4 & 33.5 \\
\hline 68 & 34.0 & 77.9 & 34.0 & 41.0 & 34.0 \\
\hline 69 & 34.5 & 79.1 & 34.5 & 41.6 & 34.5 \\
\hline 70 & 35.0 & 80.2 & 35.0 & 42.2 & 35.0 \\
\hline 71 & 35.5 & 81.3 & 35.5 & 42.8 & 35.5 \\
\hline 72 & 36.0 & 82.5 & 36.0 & 43.4 & 36.0 \\
\hline 73 & 36.5 & 83.6 & 36.5 & 44.0 & 36.5 \\
\hline 74 & 37.0 & 84.8 & 37.0 & 44.6 & 37.0 \\
\hline 75 & 37.5 & 85.9 & 37.5 & 45.2 & 37.5 \\
\hline 76 & 38.0 & 87.1 & 38.0 & 45.8 & 38.0 \\
\hline 77 & 38.5 & 88.2 & 38.5 & 46.4 & 38.5 \\
\hline 78 & 39.0 & 89.4 & 39.0 & 47.0 & 39.0 \\
\hline 79 & 39.5 & 90.5 & 39.5 & 47.6 & 39.5 \\
\hline 80 & 40.0 & 91.7 & 40.0 & 48.2 & 40.0 \\
\hline 81 & 40.5 & 92.8 & 40.5 & 48.8 & 40.5 \\
\hline 82 & 41.0 & 94.0 & 41.0 & 49.4 & 41.0 \\
\hline 83 & 41.5 & 95.1 & 41.5 & 50.0 & 41.5 \\
\hline 84 & 42.0 & 96.2 & 42.0 & 50.6 & 42.0 \\
\hline 85 & 42.5 & 97.4 & 42.5 & 51.2 & 42.5 \\
\hline 86 & 43.0 & 98.5 & 43.0 & 51.8 & 43.0 \\
\hline 87 & 43.5 & 99.7 & 43.5 & 52.4 & 43.5 \\
\hline 88 & 44.0 & 100.8 & 44.0 & 53.0 & 44.0 \\
\hline 89 & 44.5 & 102.0 & 44.5 & 53.6 & 44.5 \\
\hline 90 & 45.0 & 103.1 & 45.0 & 54.2 & 45.0 \\
\hline
\end{tabular}




\begin{tabular}{|c|c|c|c|c|c|}
\hline 91 & 45.5 & 104.3 & 45.5 & 54.8 & 45.5 \\
\hline 92 & 46.0 & 105.4 & 46.0 & 55.4 & 46.0 \\
\hline 93 & 46.5 & 106.6 & 46.5 & 56.0 & 46.5 \\
\hline 94 & 47.0 & 107.7 & 47.0 & 56.6 & 47.0 \\
\hline 95 & 47.5 & 108.8 & 47.5 & 57.2 & 47.5 \\
\hline 96 & 48.0 & 110.0 & 48.0 & 57.8 & 48.0 \\
\hline 97 & 48.5 & 111.1 & 48.5 & 58.4 & 48.5 \\
\hline 98 & 49.0 & 112.3 & 49.0 & 59.0 & 49.0 \\
\hline 99 & 49.5 & 113.4 & 49.5 & 59.6 & 49.5 \\
\hline 100 & 50.0 & 114.6 & 50.0 & 60.2 & 50.0 \\
\hline 101 & 50.5 & 115.7 & 50.5 & 60.8 & 50.5 \\
\hline 102 & 51.0 & 116.9 & 51.0 & 61.4 & 51.0 \\
\hline 103 & 51.5 & 118.0 & 51.5 & 62.0 & 51.5 \\
\hline 104 & 52.0 & 119.2 & 52.0 & 62.6 & 52.0 \\
\hline 105 & 52.5 & 120.3 & 52.5 & 63.2 & 52.5 \\
\hline 106 & 53.0 & 121.4 & 53.0 & 63.8 & 53.0 \\
\hline 107 & 53.5 & 122.6 & 53.5 & 64.4 & 53.5 \\
\hline 108 & 54.0 & 123.7 & 54.0 & 65.0 & 54.0 \\
\hline 109 & 54.5 & 124.9 & 54.5 & 65.6 & 54.5 \\
\hline 110 & 55.0 & 126.0 & 55.0 & 66.2 & 55.0 \\
\hline 111 & 55.5 & 127.2 & 55.5 & 66.9 & 55.5 \\
\hline 112 & 56.0 & 128.3 & 56.0 & 67.5 & 56.0 \\
\hline 113 & 56.5 & 129.5 & 56.5 & 68.1 & 56.5 \\
\hline 114 & 57.0 & 130.6 & 57.0 & 68.7 & 57.0 \\
\hline 115 & 57.5 & 131.8 & 57.5 & 69.3 & 57.5 \\
\hline 116 & 58.0 & 132.9 & 58.0 & 69.9 & 58.0 \\
\hline 117 & 58.5 & 134.1 & 58.5 & 70.5 & 58.5 \\
\hline 118 & 59.0 & 135.2 & 59.0 & 71.1 & 59.0 \\
\hline 119 & 59.5 & 136.3 & 59.5 & 71.7 & 59.5 \\
\hline 120 & 60.0 & 137.5 & 60.0 & 72.3 & 60.0 \\
\hline 121 & 60.5 & 138.6 & 60.5 & 72.9 & 60.5 \\
\hline 122 & 61.0 & 139.8 & 61.0 & 73.5 & 61.0 \\
\hline 123 & 61.5 & 140.9 & 61.5 & 74.1 & 61.5 \\
\hline 124 & 62.0 & 142.1 & 62.0 & 74.7 & 62.0 \\
\hline 125 & 62.5 & 143.2 & 62.5 & 75.3 & 62.5 \\
\hline 126 & 63.0 & 144.4 & 63.0 & 75.9 & 63.0 \\
\hline 127 & 63.5 & 145.5 & 63.5 & 76.5 & 63.5 \\
\hline 128 & 64.0 & 146.7 & 64.0 & 77.1 & 64.0 \\
\hline 129 & 64.5 & 147.8 & 64.5 & 77.7 & 64.5 \\
\hline 130 & 65.0 & 148.9 & 65.0 & 78.3 & 65.0 \\
\hline 131 & 65.5 & 150.1 & 65.5 & 78.9 & 65.5 \\
\hline 132 & 66.0 & 151.2 & 66.0 & 79.5 & 66.0 \\
\hline 133 & 66.5 & 152.4 & 66.5 & 80.1 & 66.5 \\
\hline 134 & 67.0 & 153.5 & 67.0 & 80.7 & 67.0 \\
\hline 135 & 67.5 & 154.7 & 67.5 & 81.3 & 67.5 \\
\hline 136 & 68.0 & 155.8 & 68.0 & 81.9 & 68.0 \\
\hline
\end{tabular}




\begin{tabular}{|llllll|}
137 & 68.5 & 157.0 & 68.5 & 82.5 & 68.5 \\
138 & 69.0 & 158.1 & 69.0 & 83.1 & 69.0 \\
139 & 69.5 & 159.3 & 69.5 & 83.7 & 69.5 \\
140 & 70.0 & 160.4 & 70.0 & 84.3 & 70.0 \\
141 & 70.5 & 161.5 & 70.5 & 84.9 & 70.5 \\
142 & 71.0 & 162.7 & 71.0 & 85.5 & 71.0 \\
143 & 71.5 & 163.8 & 71.5 & 86.1 & 71.5 \\
144 & 72.0 & 165.0 & 72.0 & 86.7 & 72.0 \\
145 & 72.5 & 166.1 & 72.5 & 87.3 & 72.9 \\
146 & 73.0 & 167.3 & 73.0 & 88.5 & 73.0 \\
147 & 73.5 & 168.4 & 73.5 & 89.1 & 73.5 \\
149 & 74.0 & 169.6 & 74.0 & 89.7 & 74.0 \\
150 & 74.5 & 170.7 & 74.5 & 90.3 & 75.0 \\
\hline
\end{tabular}

